\title{
Criminologie
}

\section{Le renvoi et la classification des infractions d'agression sexuelle}

\section{Julian Roberts et Alvaro Pires}

Volume 25, numéro 1, 1992

Les femmes et le contrôle pénal, questions féministes

URI : https://id.erudit.org/iderudit/017314ar

DOI : https://doi.org/10.7202/017314ar

Aller au sommaire du numéro

Éditeur(s)

Les Presses de l'Université de Montréal

ISSN

0316-0041 (imprimé)

1492-1367 (numérique)

Découvrir la revue

Citer cet article

Roberts, J. \& Pires, A. (1992). Le renvoi et la classification des infractions d'agression sexuelle. Criminologie, 25(1), 27-63.

https://doi.org/10.7202/017314ar
Résumé de l'article

This study is a theoretical and empirical analysis of the new tripartite structure of sexual offences created by the Criminal code reform of 1983 in Canada (Bill C-127). The authors analyze the reform proposals advanced by the Law Reform Commission as well as the data on reports of sexual assault in Quebec and Canada as a whole. In addition, the authors explore the actual classification practices of the criminal justice system and some of the "new " symbolic effects of the legislation. 


\section{LE RENVOI ET LA CLASSIFICATION DES INFRACTIONS D'AGRESSION SEXUELLE ${ }^{1}$ Julian Roberts ${ }^{2}$ et Alvaro P. Pires ${ }^{3}$}

This study is a theoretical and empirical analysis of the new tripartite structure of sexual offences created by the Criminal code reform of 1983 in Canada (Bill C-127). The authors analyze the reform proposals advanced by the Law Reform Commission as well as the data on reports of sexual assault in Quebec and Canada as a whole. In addition, the authors explore the actual classification practices of the criminal justice system and some of the "new" symbolic effects of the legislation.

Il y a un grand nombre de questions importantes pour les femmes concernant la réforme du système pénal. Ces questions concernent aussi bien les femmes victimes que les femmes justiciables. Cependant, depuis les années 1970 , on a particulièrement visé les infractions d'agression sexuelle en raison peut-être de leur signification dans le cadre des rapports de pouvoir entre les hommes et les femmes. Dans cette étude. nous ferons une analyse théorique et empirique de quelques aspects de la réforme législative de 1983 au Canada concernant les agressions sexuelles.

Cette étude vise plus spécifiquement à attirer l'attention sur deux aspects de cette énorme question. D'une part, nous voulons présenter et discuter quelques données récentes sur le taux de renvoi en matière d'agression sexuelle et, d'autre part, nous voulons analyser la nouvelle structure tripartite d'infractions créée par la réforme de 1983. Du même coup, nous voulons aussi rappeler quelques ambivalences qui ont caractérisé les divers discours et propositions de réforme. Ces ambivalences reflètent les représentations divergentes que l'on se fait de la valeur symbolique et des effets pratiques du droit criminel dans la résolution

1. Nous remercions le ministère du Solliciteur général du Canada pour le soutien financier apporté à ce projet. Nous remercions aussi la section de recherche du ministère de la Justice du Canada pour nous avoir facilité l'accès aux données et documents nécessaires. Enfin, nous voulons aussi remercier les lecteurs anonymes pour leurs suggestions à la première version de cette étude.

2. Professeur, département de criminologie, Université d'Ottawa, Pavillon Tabaret, Ottawa (Ontario), K1N 6N5.

3. Directeur, département de criminologie, Université d'Ottawa, Pavillon Tabaret, Ottawa (Ontario), K1N 6N5. 
des problèmes sociaux. Elles relèvent aussi d'un dilemme de fond concernant la place que chaque personne ou groupe juge devoir accorder au droit criminel dans le cadre d'une réponse à ces problèmes et concernant le type de droit (criminel) que l'on souhaite avoir (plus répressif ou plus axé sur la modération, etc.).

\section{LES NOUVELLES INFRACTIONS D'AGRESSION SEXUELLE}

Nous ne pouvons pas donner ici une vue générale de la réforme de 1983. Qu'il suffise de dire, pour notre propos, que le projet de loi C-127 a modifié, entre autres choses, différentes infractions sexuelles du Code criminel du Canada. Les principaux enjeux autour de cette réforme ont déjà fait l'objet d'un nombre considérable d'études, grâce notamment aux recherches féministes (Cohen et Backhouse, 1980 ; Pickard, 1980 ; Macdonald, 1982a ; 1982b ; Chase, 1983 ; Boyle, 1984 ; 1985 ; Hinch, 1985 ; 1988 ; Snider, 1985 ; Boyd et Sheehy, 1986 : Renner et Sahjpaul, 1986 ; Dawson, 1987 ; Minch, Linden et Johnson, 1987 ; Sheehy, 1987 ; Los, 1990a ; 1990b). Rappelons que la réforme canadienne s'inscrit dans le cadre d'un mouvement de portée internationale qui a pris forme tant dans les pays du common law que dans les pays suivant la tradition $\mathrm{du}$ droit romain. Nous nous limiterons à rappeler quelques grandes lignes de cette réforme en ce qui concerne spécifiquement quatre (anciennes) infractions: les deux variantes de l'attentat à la pudeur (art. 149 et 156), la tentative de viol (art. 145) et le viol (art. 143 et 144).

Les quatre infractions ci-haut mentionnées ont été remplacées par trois nouvelles infractions: (i) l'agression sexuelle (art. 271); (ii) l'agression sexuelle armée, avec menaces à une tierce personne ou avec infliction de lésions corporelles; (iii) l'agression sexuelle grave. Le tableau 1 présente un résumé des infractions avant et après la réforme de 1983 .

Il faut garder à l'esprit gu'il n'y a pas à proprement parler de correspondance juridique directe entre les comportements incriminés par chacune des quatre infractions abolies et ceux incriminés par chacune des trois nouvelles infractions. En effet, les comportements sont distribués différemment dans ces nouvelles infractions. Ainsi, un comportement qui aurait été classé comme viol avant la réforme de 1983 peut être classé dans une des trois nouvelles infractions d'agression sexuelle selon les circonstances de l'événement (et les pratiques judiciaires). Cela veut dire que l'on ne peut pas supposer, par exemple, que 
Tableau 1

Comparaison entre quelques infractions sexuelles avant et après la réforme de 1983

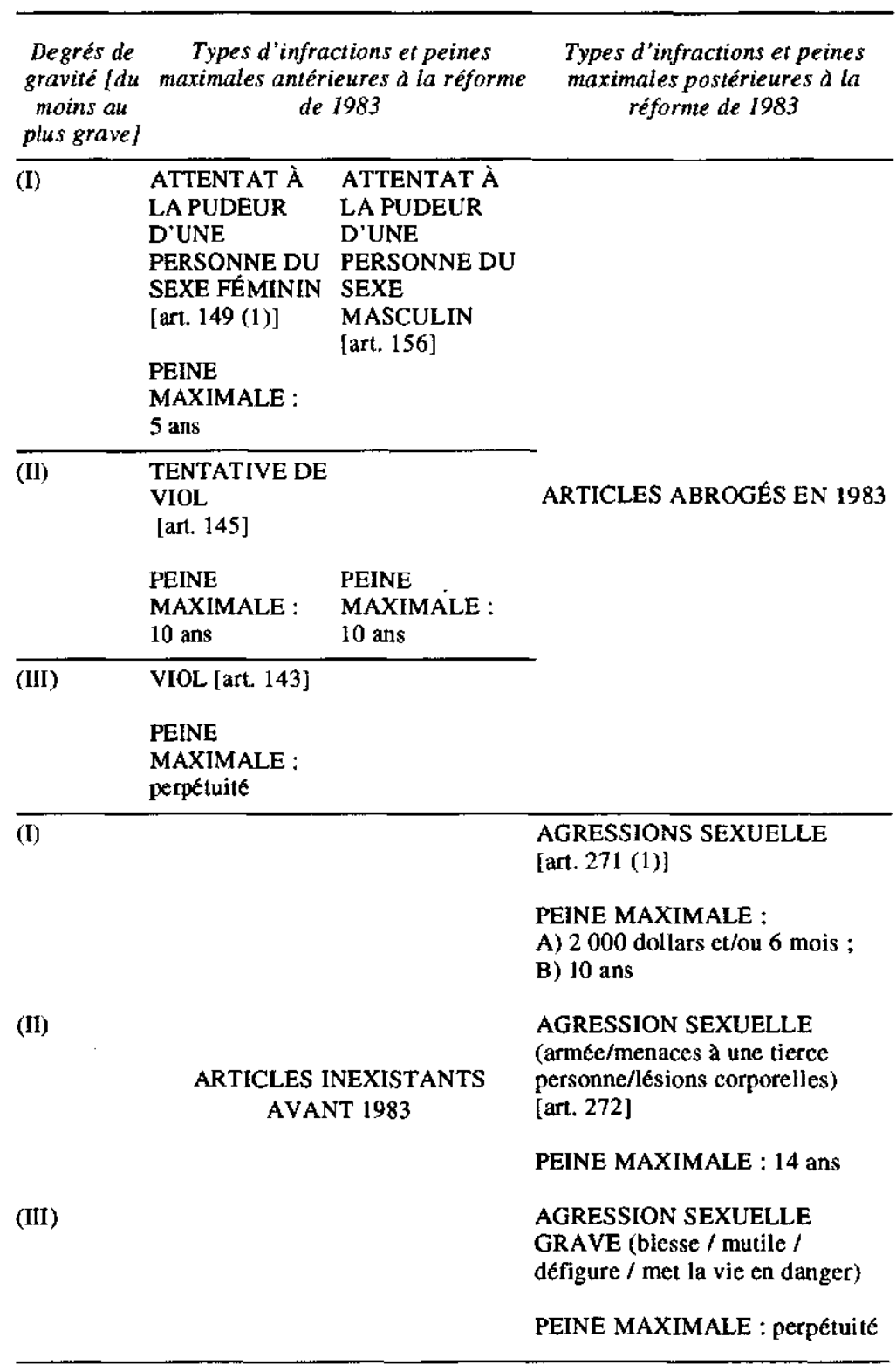


l'infraction de viol (avant 1983) et l'infraction d'agression sexuelle grave (après 1983) comprennent, du point de vue juridique, un « ensemble comparable » de comportements. Cependant, il semble que les deux groupes d'infractions (les quatre infractions avant 1983 et les trois infractions après 1983) gardent une certaine homologie dans leur ensemble.

Si les nouvelles infractions réorganisent autrement les différentes formes de contentieux, il est important cependant de noter qu'elles ont conservé, en la rendant encore plus visible, l'ancienne "échelle de gravité » en trois paliers. Pour l'instant, disons qu'avant la réforme l'image de l'échelle de gravité ressortait fondamentalement des peines maximales. Ainsi, l'infraction d'attentat à la pudeur d'une personne du sexe féminin correspondait en termes de peine maximale ( 5 ans) au premier palier de l'échelle de gravité (le «moins grave»). La tentative de viol correspondait au palier intermédiaire (10 ans) et le viol, au troisième palier (perpétuité). La classification de l'ancienne infraction d'attentat à la pudeur contre une personne du sexe masculin pose un problème particulier. En effet, en termes de définition des comportements, elle est l'équivalent de l'attentat à la pudeur d'une personne du sexe féminin et on l'associe au premier palier de gravité. Cependant, la peine maximale prévue ici est de 10 ans. Sous cet angle, cette infraction correspond plutôt au palier intermédiaire. En effet, elle crée une "réserve de pouvoir » équivalente à celle créée par l'infraction de tentative de viol (10 ans), apparemment pour compenser le fait qu'elle est la seule infraction de ce groupe applicable à une victime du sexe masculin.

Sur le plan formel et symbolique du Code criminel, nous pouvons donc constater que la réforme de 1983 a modifié et réorganisé la structure interne de chacun des trois paliers de l'échelle de gravité en fonction de nouvelles définitions et de nouvelles peines maximales. La première catégorie est devenue alors une infraction hybride ${ }^{4}$ où la peine maximale pour l'acte criminel est passée de 5 à 10 ans; la deuxième catégorie est passée d'un emprisonnement de 10 à 14 ans; puis on a retenu la peine maximale de perpétuité pour la troisième. Il n'est pas facile de décoder les raisons, les significations et les conséquences actuelles et futures de cette modification dans le système de classification et dans l'apparente élévation du seuil des peines maximales.

4. L'infraction hybride prévoit deux modes de poursuite : elle peut être poursuivie soit par voic d'une déclaration sommaire de culpabilité, soit comme un acte criminel. 


\section{LES PROPOSITIONS DE LA COMMISSION DE RÉFORME ET LE PROJET DE LOI C-127}

La Commission de réforme du droit du Canada, créée en 1971, constitue un point important de ce processus de réforme. Nous examinerons ici ses propositions parce qu'elles ont été, entre autres choses, moins discutées dans la littérature et parce qu'elles ont pris la forme d'un véritable projet en ce qui concerne la définition des infractions et le choix des peines maximales. Cependant, pour bien comprendre certains aspects de ce projet, il faut le situer dans le sillage des mouvements féministes à la fin des années 1960 et au début des années 1970 (Snider, 1985; Los, 1990a ; 1990b). Ces mouvements ont mis alors sur le tapis la question de la violence faite aux femmes et ont dénoncé les biais sexistes que l'on trouvait dans les lois et dans les pratiques de la justice. En appliquant aux agressions sexuelles les remarques plus générales de Smart $(1989$, p. 21$)$, on peut dire qu'au début les auteures féministes se sont concentrées sur trois questions majeures : on a critiqué le droit criminel pour avoir exclu les femmes du champ de la protection juridique; on a critiqué les biais sexistes dans la définition même du viol et on a critiqué des pratiques spécifiques de la police et des tribunaux en cette matière.

Le document de travail de la C.R.D.C. (1978a) fait état aussi de trois préoccupations majeures dont deux au moins ont été soulevées par le mouvement féministe. Tout d'abord, il affirme que les statistiques pénales accusent une augmentation dans le nombre des contentieux soumis à la justice. La commission remarque que l'on ne peut pas savoir s'il s'agit là d'une augmentation des actes d'agression, d'une propension plus grande à porter plainte ou d'une conjugaison de ces deux facteurs. Quoi qu'il en soit, le message ici est le suivant: il faut absolument que le droit criminel s'occupe de ce problème de manière plus adéquate et conforme à nos valeurs actuelles.

Le deuxième point fut présenté sous une double facette. D'une part, on reconnaît la nécessité de modifier la définition même de viol et, d'autre part, on propose de le considérer comme une variété de voies de fait pour mettre l'accent sur la dimension de violence et d'atteinte à l'intégrité de la personne (par voie d'attentat sexuel) plutôt que sur l'aspect sexuel lui-même (C.R.D.C., 1978a, pp. 15-18). Bref, on veut considérer le viol comme un acte d'agression avec une dimension sexuelle et non pas comme un «acte sexuel illégal " (p. 23). Ce faisant, la commission faisait écho aux revendications de la première vague 
d'études féministes qui voulait « dé-sexualiser » le viol (Griffin, 1971; Russell, 1975: Clark et Lewis, 1977) $)^{5}$. Les anciennes infractions étaient placées alors dans la partie IV du Code intitulée «infractions d'ordre sexuel, actes contraires aux bonnes mœurs, inconduite». Il s'agissait donc de les placer dans la partie appelée « infractions contre la personne et la réputation ».

Enfin, eu égard au troisième point, la commission indiquait clairement que l'enquête et le procès sont devenus des «sources grandissantes de préoccupation ». Le document déplore ici la situation pénible de la femme victime de viol devant la justice criminelle.

La définition du viol, quant à elle, faisait principalement l'objet de deux modifications. Afin de correspondre à une conception plus « égalitaire" du droit, la distinction entre les femmes et les hommes devait être éliminée (C.R.D.C., 1978a, pp. 16-17). L'agression sexuelle devait être définie de manière à désigner tant les femmes que les hommes, sans faire la différence entre le sexe de la victime et celui de l'agresseur. Puis, afin d'élargir la protection juridique à l'égard des femmes, la définition devait aussi pouvoir s'appliquer à la femme mariée pour ne pas entériner le principe selon lequel le mari aurait des droits sexuels sur son épouse sans le consentement de celle-ci (pp. 17-18). Elle devait ensuite éliminer l'exigence de la pénétration du pénis dans le vagin comprise dans la définition en vigueur à l'époque. On voulait ici notamment inclure les cas de pénétration orale ou anale.

Dans ce document, la commission (1978a) a recommandé qu'il y ait seulement une infraction d'agression sexuelle et n'a fait aucune recommandation concernant la peine maximale ou la création d'une infraction hybride. Pour la commission, l'infraction d'agression sexuelle ne devait être alors divisée ni en fonction de comportements différents (viol, attentat à la pudeur, etc.), ni en fonction d'une échelle légale de gravité (agressions sexuelles plus ou moins graves). Elle a cependant proposé que le tribunal tienne compte, au niveau de la sentence. de toutes les circonstances et conséquences de la situation, y compris la pénétration ou la violence.

Un peu plus tard, dans son rapport au ministre de la Justice, la commission (1978b, p. 14) a modifié sa position, suite à certaines critiques, et a proposé que les infractions de viol, de tentative de viol,

5. Pour une révision de ce débat dans les écrits féministes et par rapport à Foucault, voir l'excellent article de Bell (1991). 
Tableau 2

Trois propositions de réforme des infractions sexuelles au Canada (1978-1983)

\begin{tabular}{clc}
$\begin{array}{c}\text { Première proposition de la } \\
\begin{array}{c}\text { Commission de réforme } \\
\text { Document de travail } \\
(1978 a)\end{array}\end{array}$ & $\begin{array}{l}\text { Deuxieme proposition de } \\
\text { la Commission de réforme } \\
\text { Rapport au ministre de la } \\
\text { Justice }(1978 b)\end{array}$ & $\begin{array}{c}\text { Version finale du projet } \\
\text { C-127 }\end{array}$ \\
\hline $\begin{array}{l}\text { Infraction unique } \\
\text { * Attentat sexuel * }\end{array}$ & $\begin{array}{l}\text { Structure bipartite } \\
\text { I) * Attouchement } \\
\text { sexuel * }\end{array}$ & $\begin{array}{l}\text { Structure tripartite } \\
\text { I) * Agression sexuelle * }\end{array}$
\end{tabular}

Toute personne qui a un Quiconque, dans un but Quiconque commet une contact sexuel avec autrui, sexuel, touche directement agression sexuelle est sans son consentement, est ou indirectement une autre coupable : coupable de 1'infraction personne sans le d'attentat sexuel. consentement de cette dernière est coupable : Peine maximale : non $\quad$ a) d'un acte criminel et proposée passible d'un emprisonnement de cinq ans, ou

a) soit d'un acte criminel et passible d'un emprisonnement maximal de dix ans ;

b) d'une infraction punis- b) soit d'une infraction sable sur déclaration punissable sur déclaration sommaire de culpabilité. de culpabilité par procé[Peine maximale : six mois dure sommaire. [Peine et/ou 2000 dollars maximale : six mois et/ou d'amende]. 2000 dollars d'amende.]

(A) L'expression « contact II) * Agression sexuelle * II) * Agression sexuelle sexuel " dans le présent. article comprend tout attouchement non accidentel des organes sexuels d'autrui ou le fait non accidentel pour une personne d'en toucher une autre avec ses propres organes sexuels, et d'une façon qui porte atteinte à la dignité sexuelle de cette personne.

Quiconque emploie ou menace d'employer la violence dans la perpétration d'un attouchement sexuel est coupable d'un acte criminel et passible d'un emprisonnement de dix ans.
(B) En imposant une sentence à une personne condamnée en vertu du présent article, le tribunal doit tenir compte de toutes les circonstances et conséquences de l'attentat, $y$ compris du fait qu'il y a eu pénétration ou violence. armée, menace à tierce personne ou infliction de lésions corporelles 》

Est coupable d'un acte criminel et passible d'un emprisonnement maximal de quatorze ans quiconque, en commettant une agression sexuelle, selon le cas :

a) porte, utilise ou menace d'utiliser une arme ou une imitation d'arme :

b) menace $d$ infliger des lésions corporelles à une autre personne que le plaignant ;

c) inflige des lésions au plaignant ;

d) participe a l'infraction avec une autre personne. 


\begin{tabular}{|c|c|c|}
\hline $\begin{array}{c}\text { Première proposition de la } \\
\text { Commission de réforme } \\
\text { Document de travail } \\
(1978 a)\end{array}$ & $\begin{array}{l}\text { Deuxieme proposition de } \\
\text { la Commission de réforme } \\
\text { Rapport au ministre de la } \\
\text { Justice (1978b) }\end{array}$ & $\begin{array}{l}\text { Version finale du projet } \\
\text { C-127 } \\
\text { Réforme de } 1983\end{array}$ \\
\hline & & $\begin{array}{l}\text { III) * Agression sexuelle } \\
\text { grave " } \\
\text { Commet une agression } \\
\text { sexuelle grave quiconque, } \\
\text { en commettant une } \\
\text { agression sexuelle, blesse, } \\
\text { mutile ou defigure le plai- } \\
\text { gnant ou met sa vie en } \\
\text { danger. }\end{array}$ \\
\hline
\end{tabular}

d'attentat à la pudeur et de grossière indécence (art. 157) soient abolies et remplacées par deux nouvelles infractions : l'infraction d' " attouchement sexuel » (sexual interference) et celle d' «agression sexuelle». La commission voulait alors souligner, au plan même de la définition de l'infraction (et non seulement au plan de la sentence), le caractère aggravant du recours à la violence (physique). En effet, la commission distinguait le "simple attouchement sexuel non accompagné de violence ou de menaces d'un côté ; d'un autre côté l'attentat sexuel accompagné de violence ou de menaces» (p. 15). En outre, elle affirmait que cette distinction entre "le simple attouchement sexuel et l'agression sexuelle n'est pas seulement une de degré, mais bien plus une d'espèce " (p. 15). C'est alors qu'elle a proposé aussi pour la première fois que l'infraction d' " attouchement sexuel » soit une infraction hybride. La peine maximale pour la procédure sommaire serait, bien sûr, celle prévue dans le Code criminel pour ce mode de poursuite (six mois d'emprisonnement et/ou 2000 dollars d'amende); pour l'acte criminel, la peine maximale proposée a été de 5 ans. La deuxième infraction, celle d' "agression sexuelle», devait être considérée comme un acte criminel et être passible d'un emprisonnement maximal de 10 ans.

Cette peine maximale de 10 ans proposée par la commission reflétait sa préoccupation avec le principe de modération mais, en termes pratiques, elle n'était pas à proprement parler très avant-gardiste. En effet, comme la structure des peines de notre code date au moins du XIXe siècle, les peines à perpétuité sont devenues anachroniques et trompeuses, puisqu'elles ne correspondent plus à la pratique effective des tribunaux (voir C.C.D.P., 1987, pp. 68-70, 214-231). Ainsi, une recherche faite à Montréal nous indique que 96 pour cent des cas 
d'agression sexuelle que sanctionne une peine d'emprisonnement ont une durée de moins de 10 ans, et ce autant avant la réforme (1981 et 1982) qu'après (1984 et 1985) (Baril, Bettez et Viau, 1988, p. 184) ${ }^{6}$. On peut donc dire que la proposition de la commission « actualisait » tout simplement le code par rapport aux pratiques actuelles. Cependant, cette proposition impliquait un choix difficile pour certaines personnes. C'est qu'il existe un nombre énorme de peines maximales anachroniques dans le code et l'acceptation de cette proposition provoquerait un contraste avec les autres peines. Par exemple, le vol qualifié et l'introduction par effraction dans une maison d'habitation sont aussi encore frappés d'une peine maximale d'emprisonnement à perpétuité. Si l'on modifie alors la peine maximale pour les agressions sexuelles, à un moment où l'on veut exprimer une réprobation morale à l'égard de ces situations, sans modifier en même temps les autres peines maximales désuètes ou aberrantes, le message symbolique de la loi serait, de l'avis de certains, ambigu. Voilà donc le dilemme : si l'on tient à la cohérence ou si l'on insiste sur les effets symboliques supposés de la peine, on sacrifie la modération; si l'on privilégie la modération, on doit faire des concessions sur l'importance que nous attribuons à la cohérence et aux effets symboliques supposés des peines.

Comme nous pouvons le voir au tableau 1 , le projet de loi C-127 a modifié considérablement les propositions de la commission. D'abord, les recommandations de celle-ci touchant les peines maximales n'ont pas survécu au processus de réforme. Les peines ont été clairement augmentées par rapport aux propositions qu'a faites la commission. Certes, il y a cependant un doute sur la manière d'interpréter ces changements par rapport aux anciennes peines maximales, particulièrement celle de l'attentat à la pudeur d'une personne du sexe féminin (5 ans). Deuxièmement, on a crế trois niveaux d'infractions au lieu de deux. Troisièmement, on a décidé de donner la même appellation d' «agression sexuelle" aux trois infractions et de ne pas faire de distinction conceptuelle entre ces infractions. L'une des conséquences de ce choix est que la distinction d'espèce entre l'attouchement sexuel et l'agression sexuelle disparaît sur le plan de la classification et de la clarification juridique des situations. Quatrièmement, on décide de ne pas définir explicitement la première infraction d'agression sexuelle (voir

6. Selon les auteures, la durée des peines d'emprisonnement est đemeurếe la même avant et après la réforme pour les années considérées. Toutefois, le nombre de sentences d'emprisonnement a augmenté (p. 184). 
tableau 2) ${ }^{7}$. L'appellation «agression sexuelle" au premier niveau semble prendre alors une acception plus large et plus vague que celle donnée par la deuxième proposition de la Commission de réforme. Car, à première vue, ce premier niveau peut inclure certaines formes moindres de violence physique. Le deuxième niveau vaut pour les situations où l'agresseur: (i) porte, utilise ou menace d'utiliser une arme; (ii) menace d'infliger des lésions corporelles à une tierce personne; (iii) inflige des lésions corporelles à sa victime; (iv) ou participe à l'infraction avec une autre personne. Le troisième niveau, appelé "agression sexuelle grave", s'applique aux cas où l'agresseur blesse, mutile ou défigure sa victime ou met sa vie en danger. On perçoit alors d'emblée trois difficultés d'interprétation, tout particulièrement pour le sens commun : celles reliées au manque de définition du premier niveau, à la notion de lésions corporelles au deuxième niveau (C.R.D.C., 1984) et à la notion de «blessure » au troisième niveau.

Voyons sommairement l'exercice que les tribunaux sont appelés à faire. La notion de "lésions corporelles " est l'un des critères pour classifier certaines situations avec une composante de violence physique dans la deuxième catégorie. L'article 267 (2) du Code criminel dit que cette expression doit désigner « une blessure qui nuit à la santé ou au bien-être du plaignant et qui n'est pas de nature passagère ou sans importance ". Les tribunaux doivent donc séparer la violence physique qui appartient au premier niveau des lésions corporelles qui appartiennent au deuxième niveau et ces dernières de la blessure et de la mutilation qui font partie du troisième niveau. Tout se passe alors comme si on avait voulu diviser en trois la "violence physique" des agressions sexuelles, sans élucider les critères de ce partage. L'utilisation d'une arme apparaît alors comme le seul critère de tout repos dans ce nouveau système de classification. On peut dire que la réforme de 1983 a créé une certaine confusion d'interprétation autour de ces trois niveaux d'infractions.

Enfin, remarquons aussi que dans l'ensemble on a mis encore plus en évidence l'image selon laquelle ces infractions désignent fondamentalement une «échelle de gravité ». Une autre confusion s'est glissée alors. En effet, que mesure cette échelle exactement ? Est-elle une échelle de violence physique ou, au contraire, une échelle purement

7. L'article 265 (1) et (2) donne une sorte de définition générale de " voies de fail " et d' * agression » qui sert de toile de fond, entre autres, aux articles 266 (premier niveau de voies de fait) et 271 (1) (premier niveau d'agression sexuelle) où une définition explicite fait clairement défaut. 
symbolique de réprobation morale ? Autrement dit, quelle est la fonction première de cette échelle ? Doit-elle servir avant tout de guide à l'intention des juges pour punir plus sévèrement les situations les plus violentes, ou doit-elle servir plutôt à indiquer grossièrement au grand public la gravité relative de ces infractions par rapport à d'autres ? C'est la dernière hypothèse qui paraît la plus vraisemblable. Car il est difficile de supposer qu'en prévoyant une peine aussi élevée (10 ans) pour le niveau le moins grave, le « législateur » ait « voulu » pousser les tribunaux à augmenter aussi dramatiquement leurs sentences. Il est plus raisonnable de croire que le «législateur» ait tout simplement « voulu » exprimer par là l'importance symbolique relative de ces infractions dans le code actuel. Bien sûr, ce choix risque, entre autres choses, de créer un quiproquo au niveau des attentes créées par la loi et des différentes perceptions que l'on peut avoir de ce changement. De plus, en prévoyant une peine maximale aussi élevée pour le premier niveau, on donne aux tribunaux la possibilité d'y traiter « confortablement » (du point de vue de la détermination des peines) de la quasitotalité des cas d'agression sexuelle.

\section{QUESTIONS OUVERTES SUR LES AMBIVALENCES DANS L'ORIENTATION DE LA RÉFORME}

Il convient maintenant d'indiquer, à titre exploratoire, quelques tendances contradictoires et ambivalences qui existaient dans les orientations philosophiques et sociales de la réforme de 1983 par rapport au caractère plus ou moins «répressif» ou, au contraire, plus ou moins « modérê » que devrait prendre la nouvelle loi.

Particulièrement dans les documents publiés au cours des années 1970, la Commission de réforme du droit demandait l'adoption d'une politique de réforme axée sur le principe de la modération dans le recours aux tribunaux, dans l'imposition des sanctions, voire dans le recours au droit criminel tout court comme méthode pour réagir aux problèmes sociaux. L'orientation philosophique générale de la commission tendait alors à un droit criminel minimal qui, en principe, n'interviendrait dans la vie des gens que dans la mesure où cela s'avérerait absolument nécessaire. Elle recommandait de restreindre la portée de la notion d' «infraction criminelle», de réduire le nombre d'infractions, de réduire l'échelle globale des peines maximales d'emprisonnement prévues par le Code criminel, etc. Elle demandait aussi de rénover le langage utilisé et de définir de façon claire les infractions afin de rendre le droit compréhensible et accessible au public en général (C.R.D.C., 
1978a, p. 6), au lieu de donner l'impression que les règles de droit s'adressent principalement au personnel de la justice. Comme nous l'avons vu, la peine maximale de 10 ans proposée par la commission (1978b) pour l'infraction d'agression sexuelle reflète cette préoccupation avec modération, car elle réduit l'ancienne peine maximale pour viol (perpétuité). En revanche, la peine de 5 ans pour l'infraction d'attouchement sexuel (sans menace et sans violence physique) paraît moins marquée au coin de la modération. En effet, rappelons que l'ancienne infraction hybride pour voies de fait avec lésions corporelles (art. 245 (2), C.cr. 1975) avait justement une peine maximale de cinq ans. Il s'agit donc d'une peine relativement sévère.

Dans son document de travail, la commission (1978a) a d'abord rappelé que le droit pénal devrait « favoriser au maximum la liberté des personnes et réduire au minimum l'intervention de l'État, à cause de ses effets possibles sur la personne et sur l'État, en cas d'usage abusif ou immodéré " (p. 4). Dès lors « le recours au droit pénal comme moyen de contrôle social est un recours de dernier ressort $»(p .4)$. Elle a soutenu que le droit pénal a «ses propres limites comme méthode de contrôle social » et qu'il ne doit pas être envisagé «comme le seul moyen qu'a la société pour réagir aux problèmes sociaux » (p. 7). Pour la commission, "qu'il ait été rigoureux ou clément, le droit pénal n'a jamais réussi à éliminer le crime» et "l'important n'est pas ce que dit la loi, mais ce qu'elle fait aux gens et ce qu'elle fait pour eux» (p. 7). En outre, aux yeux de la commission, "le fait de punir le délinquant ne comporte aucun ou peu d'avantages pour la victime» (p. 7). Dès lors, la commission souligne la nécessité de prendre en ligne de compte différentes façons de résoudre ce problème: «ces solutions de rechange n'entrent pas nécessairement en conflit avec le système de justice criminelle, et peuvent intervenir soit avant, soit en même temps que le recours au processus pénal» (p. 8). Elle rappelle que les buts des sanctions pénales sont « la manifestation d'une désapprobation sociale, la réparation du tort causé, la restauration de la paix, de l'ordre et de la confiance, et l'application d'un certain degré de contrôle", puis elle remarque que les sanctions traditionnelles (amende et emprisonnement) « ne sont pas nécessairement les plus appropriées pour certaines infractions sexuelles » (p. 47). Elle indique ensuite plusieurs problemes qui découlent du recours à l'incarcération.

Par ailleurs, comme le rappelle Pitch (1985b, p. 257), la violence sexuelle venait d'accéder au statut de "problème social " à l'initiative du mouvement đe femmes qui dénonçait, à juste titre, les biais sexistes de la loi et l'exclusion des fermmes du champ de la protection juridique. 
Il y avait donc des demandes spécifiques des groupes sociaux au système de justice (Pitch, 1985b, p. 257). Comme il n'était pas question d'une réforme globale du code, toute réforme partielle se heurtait alors au problème symbolique de la comparaison entre les nouvelles peines maximales proposées et l'ancienne structure globale des peines. Si l'on veut « redonner » aux femmes la place qui leur revient dans la structure symbolique d'un vieux code criminel, c'est-à-dire "faire l'égalité " sans envisager, à court ou à long terme, une modification de l'ensemble de la logique punitive, on doit renoncer en partie à un plaidoyer pour la modération, et ce, même si l'on ne veut pas un durcissement de la répression.

En présentant le projet de réforme du mouvement de femmes en Italie, Pitch (1985a, pp. 42-43) remarque que :

the Women's Bill does not [...] take into consideration the earlier critiques of the criminal justice system as highly selective and structurally unjust. Interviews with the promoters of the bill on this topic reveal the following types of responses: (a) we are not concerned with harsher penalties, that is not what we necessarily want: we want only the same kind of justice which has been given to other victims for other crimes; (b) this is man's "justice", we are not responsible for it : we only ask for it to be applied consistently, against other men; $(c)$ we are not interested in the details of the Bill, we want a few principles to be established, e.g. women's dignity as people, the recognition of violence to them as a major crime and $(d)$ we know that a different law will not diminish crimes and that harsher penalties will not alter the general picture: the Bill, however, is an occasion for extensive and public debate on these topics and will increase cultural and political awareness about them.

Pitch signale alors qu'il est à la fois compréhensible et assez paradoxal que cette demande sociale de justice ait été formulée à travers les présupposés du discours dominant traditionnel sur le Code criminel et que cette demande « appears to privilege its retributive and symbolic aspects, in line with a discourse which tended to be de-emphasized in the 1960s and 1970s even within the agencies of social control themselves » (Pitch, 1985a, p. 43). Los (1990a ; 1990b) a, quant à elle, fait une analyse très fine des dilemmes auxquels les groupes de femmes au Canada ont dî faire face dans le feu de l'action et compte tenu de l'état précédent des lois et surtout du procès en matière de viol. Outre les pièges posés par le discours juridique lui-même, ces groupes étaient confrontés aux représentations sociales dominantes de la justice criminelle. Or, en reprenant les termes de Pitch (1985b, p. 262), ces représen- 
tations transforment le « désir de justice » en « désir de punition », de « rétribution". On pénètre alors dans un terrain glissant où l'équation « crime-droit criminel » signifie aussi «peine-emprisonnement ». Et ce terrain est d'autant plus glissant que la réforme du droit vers la fin des années 1970 tendait à abandonner l'idée de rééducation ${ }^{8}$ et faisait un tournant vers les visées symboliques de la justice criminelle (Pitch, 1985 b, pp. 262-265). Certains voulaient que le droit criminel prenne la forme d'une dénonciation caractérisée fondamentalement par un message éthique de modération ; d'autres voulaient un droit criminel musclé et marqué par un message clairement rétributiviste ; d'autres encore voulaient surtout un droit pénal symboliquement égalitaire, dans une direction ou dans l'autre. Los constate alors que certains groupes d'intérêt des femmes, par rapport à certaines revendications, ont été «trapped in the same myopic perspective which most victim's groups seem to adopt : the victimizers were perceived as an enemy to be crushed ». Comme elle le souligne, " the desire to put rapists behind bars is more than understandable. It does not make it less myopic, however " (Los, 1990a, pp. 167-168). Bref, « the thinking about crime and punishment that permeates dominant (man-made) images of justice was thus accepted by these women who were caught between the escalating fear of rape and the paucity of alternative, non-legal avenues for action " (Los, 1990a, p. 168).

Dans ce contexte général, il n'est pas étonnant de constater que la commission n'a pas échappé non plus à certaines ambivalences et ambiguïtés. Nous allons nous interroger ici sur les raisons pour recommander à la fois la création d'une infraction bipartite et une infraction hybride.

En apparence, ces recommandations semblent particulièrement liées au principe de modération, mais en réalité cela n'est pas aussi évident. En effet, la commission affirme explicitement, en renvoyant à l'une de ses recommandations, qu'elle a été dictée par un point de vue pratique. Ces recommandations n'étaient pas comprises dans le document de travail (1978a) et semblent avoir été ajoutées suite à quelques critiques faites à ce document. Ces critiques visaient, selon toute vraisemblance, à faciliter l'intervention du droit criminel (et non à le limiter). En effet, l'objectif aurait été d'adapter le système pénal à la réception d'un plus grand nombre de situations et d'augmenter le nom-

8. Le document de travail de la Commission de réforme du droit a pressenti par ailleurs la nécessité d'affirmer la valeur des thérapies externes à la prison et même des ordonnances d'hospitalisation pour certains cas relevant de ces infractions. 
bre de condamnations en général. On redoutait que si on avait une seule infraction et que si la peine pour cette infraction était très élevée, on aurait de la difficulté à obtenir une condamnation dans les situations d'agression jugées «physiquement moins graves » par les représentations sociales dominantes ${ }^{9}$. L'enjeu ici est de savoir jusqu'à quel point on veut faire jouer le système pénal pour affirmer les valeurs auxquelles nous tenons dans le cadre d'un projet de société. Il s'agissait alors de prendre position face à la question (brûlante) de l'inclusion ou non de certaines formes d'agression dans le registre de la loi criminelle, d'une loi criminelle encore axée sur la rétribution et la prison, etc.

La commission semble avoir choisi, et par rapport aux agressions sexuelles, et par rapport aux voies de fait, une politique d'inclusion maximale tout en comptant sur le «bon sens » des gens et des opérateurs du système pour filtrer les cas les plus «triviaux». Cette option est plus claire dans le document plus tardif sur les voies de fait (C.R.D.C., 1984) que dans celui sur l'agression sexuelle. Selon la commission, cette option correspond en fait à la situation qui était déjà en vigueur. Le dilemme philosophique (et politique) est alors le suivant. Afin de justifier cette position d'inclusion maximale, la commission (1984, p. 49) soutient que si le droit criminel ne considérait pas comme un crime l'acte de « toucher intentionnellement une autre personne sans son consentement [...] ceci signifierait abandonner la notion selon laquelle les atteintes à la personne sont une valeur fondamentale ». Le dilemme est donc le suivant. Les documents de la commission affirment, d'une part, que la liberté et l'intégrité de la personne sont des valeurs si importantes que l'on doit établir clairement les limites de l'intervention étatique et particulièrement celle du droit criminel. Mais, d'autre part, ces documents soutiennent aussi que ces mêmes valeurs sont tellement importantes que, pour les défendre convenablement, il faut faire appel au droit criminel pour réagir contre toutes les formes de torts susceptibles de leur porter atteinte.

À notre avis, il est important de bien comprendre ces positions contradictoires et ambivalentes pour saisir la portée et les limites des aspects de la réforme de 1983 qui sont liés aux peines maximales et au système de classification des infractions.

9. La recherche de Los (1990a) sur le projet de loi C-127 semble confirmer cette hypothèse. 


\section{LES VISÉES DE LA RÉFORME}

Il est très difficile de préciser, de manière non équivoque, les objectifs d'un projet de réforme. En effet, ce projet fut investi par différents acteurs sociaux avec des intentionnalités et des projets différents, voire opposés (groupes féministes, Commission de réforme, représentants des procureurs, de la police, du barreau, des avocats de la défense, gouvernement lui-même, etc.) (Los, 1990, p. 163). Même les groupes de femmes ont présenté des idées et des propositions différentes (Los, 1990 , p. 163). Compte tenu de cette diversité, il serait plus juste de s'interroger séparément sur les objectifs de chacun de ces acteurs.

Selon Los (1990a, pp. 163-164), une des raisons principales qui ont poussé le gouvernement à instaurer au plus tôt une réforme a été l'inclusion, en 1982, de la Charte canadienne des droits et libertés dans la Constitution. On voulait ainsi éviter un long contentieux constitutionnel à l'égard de certains articles du Code criminel qui auraient de la difficulté à passer le test de l'égalité des droits. Cependant, ceci n'empêche pas qu'on ait aussi créé certaines attentes par rapport à la nouvelle loi. Un point, au moins, a fait l'objet d'un consensus parmi les groupes œuvrant directement dans le processus de réforme: la réforme devait entraîner une augmentation du nombre de plaintes et de condamnations dans le cadre des infractions sexuelies. Ce consensus était, certes, fragile, car il a été bâti, selon toute vraisemblance, sur des motivations fort différentes. Pour les groupes de femmes, par exemple, cet objectif devait s'accompagner fondamentalement d'un traitement moins pénible et stigmatisant pour les femmes pendant l'enquête et le jugement (Snider, 1985, pp. 343, 352 ; Los, 1990a). Nous allons examiner maintenant cette question du nombre de renvois au système de justice criminelle.

\section{LES EFFETS DE LA RÉFORME SUR LE NOMBRE DE RENVOIS}

La figure 1 présente un graphique de la fréquence des plaintes ou des situations d'agression sexuelle qui ont été renvoyées à la justice criminelle (au Québec et au Canada comme un tout) pendant les 13 années entourant le passage de la réforme de la loi. Il s'agit de situations dont la police a été saisie, à la suite de plaintes ou autrement ${ }^{10}$. Ces chiffres correspondent à ce que Statistique Canada appelle

10. Ces chiffres sont tirés du DUC (système de la déclaration uniforme de la criminalité) du Centre canadien de la statistique juridique. 
les « infractions déclarées », par opposition à ce que Statistique Canada appelle les « infractions réelles », c'est-à-dire les infractions déclarées moins celles considérées par la police comme «non fondées ». Nous avons choisi de présenter ici le nombre (plus élevé) de situations qui ont été renvoyées, mais la tendance à l'augmentation est la même dans les deux séries de données. Aussi est-il vrai que cette tendance ne se modifie pas si l'on considère plutôt le taux par cent mille habitants. Comme nous pouvons l'observer, il y a une augmentation énorme et soutenue depuis 1983. Les chiffres servant de base a la construction de ce graphique se trouvent dans le tableau 3.

Tableau 3

Nombre des renvois en matière d'agressions sexuelles au Québec et au Canada et taux de changement (en pourcentage). 1977.1989

\begin{tabular}{|c|c|c|c|c|}
\hline Années & $\begin{array}{l}\text { Québec } \\
\text { (nombre) }\end{array}$ & $\begin{array}{l}\text { Québec } \\
(\%)\end{array}$ & $\begin{array}{c}\text { Canada } \\
\text { (nombre) }\end{array}$ & $\begin{array}{c}\text { Canada } \\
(\%)\end{array}$ \\
\hline 1977 & 2161 & $\begin{array}{l}\text { Avant la } \\
\text { réforme }\end{array}$ & 10285 & $\begin{array}{l}\text { Avant la } \\
\text { réforme }\end{array}$ \\
\hline 1978 & 1982 & $-8 \%$ & 10687 & $+4 \%$ \\
\hline 1979 & 2320 & $+17 \%$ & 11557 & $+8 \%$ \\
\hline 1980 & 2313 & $0 \%$ & 12077 & $+4 \%$ \\
\hline 1981 & 2248 & $-3 \%$ & 12376 & $+2 \%$ \\
\hline $\begin{array}{l}1982 \\
1977-1982\end{array}$ & 2130 & $\begin{array}{r}-5 \% \\
(-3 \%)\end{array}$ & 12848 & $\begin{array}{r}+4 \% \\
(+25 \%)\end{array}$ \\
\hline $\begin{array}{l}1983 \\
\text { (Réforme) }\end{array}$ & 2090 & $\begin{array}{l}\text { Après la } \\
\text { réforme }\end{array}$ & 13851 & $\begin{array}{l}\text { Après la } \\
\text { réforme }\end{array}$ \\
\hline 1984 & 2495 & $+19 \%$ & 17323 & $+25 \%$ \\
\hline 1985 & 2810 & $+13 \%$ & 21264 & $+23 \%$ \\
\hline 1986 & 3267 & $+16 \%$ & 24114 & $+13 \%$ \\
\hline 1987 & 3476 & $+6 \%$ & 26443 & $+10 \%$ \\
\hline 1988 & 3778 & $+9 \%$ & 29111 & $+10 \%$ \\
\hline 1989 & 3976 & $+5 \%$ & 31756 & $+9 \%$ \\
\hline $1983-1989$ & & $(+90 \%)$ & & $(+129 \%)$ \\
\hline
\end{tabular}

N.B. : Les données avant 1983 comprennent l'ensemble des infractions de viol, de tentative de viol et d'attentat à la pudeur d'une personne du sexe féminin et masculin; les données à partir de 1983 comprennent les trois niveaux d'infractions pour les agressions sexuelles.

Examinons d'abord la situation du Québec telle que présentée dans le tableau 3. Nous constatons qu'entre 1977 et 1983, il y a eu une certaine fluctuation dans le nombre de renvois d'agression sexuelle. Les 
colonnes avec les pourcentages indiquent les variations par rapport à l'année précédente. Ainsi, au Québec, le nombre de renvois a diminué de 8 pour cent en 1978 par rapport à 1977 et a augmenté de 17 pour cent en 1979 par rapport à 1978 pour diminuer encore un peu par la suite. Cependant, non seulement la fluctuation disparaît-elle après 1984 mais les augmentations deviennent beaucoup plus marquées. Si l'on compare l'ensemble des deux périodes, il y a eu une baisse de 3 pour cent entre 1977 et 1982 et une augmentation de 90 pour cent entre 1983 et 1989. Ce dernier résultat a été sans doute exacerbé par le fait que l'année 1983 constitue la deuxième année où le nombre de renvois était à son plus bas niveau pendant toute la période observée. Mais si l'on refait les calculs en fonction de l'année 1979 (celle où le chiffre de renvoi était à son plus haut niveau avant la réforme), nous constatons quand même une augmentation de 71 pour cent entre les années 1979 et 1989. Quoi qu'il en soit, l'augmentation demeure frappante. Ces résultats coïncident d'ailleurs avec ceux de Baril, Bettez et Viau (1988, p. 58) ${ }^{11}$ qui ont trouvé, pour la région de Montréal, une augmentation de 23 pour cent entre 1981 et 1985 (nos données pour cette période sont les mêmes: 25 pour cent).

L'augmentation au Canada comme un tout est encore plus marquée. Il y a eu 25 pour cent d'augmentation entre 1977 et 1982 , mais cette augmentation a été dramatique entre 1983 et $1989: 129$ pour cent (voir tableau 3 ). Il n'y a eu aucune diminution dans les taux avant la réforme par rapport à l'année 1977, mais les augmentations ont été, toutes proportions gardées, plutôt modestes. En effet, on peut observer que le taux le plus élevé avant la réforme a été de 8 pour cent et ce pendant une seule année (1979). En revanche, le taux le plus bas après la réforme a été de 9 pour cent dans la dernière années (1989) et après une très forte augmentation immédiatement après la réforme.

Certes, il n'est pas facile d'interpréter et d'expliquer les changements observés dans une distribution d'événements dans le temps (Campbell, 1969). Cependant, lorsque ces changements débutent au moment attendu et sont marqués et soutenus, nous avons de bonnes raisons pour conserver l'hypothèse selon laquelle on peut les attribuer dans une certaine mesure à la réforme comme telle. Par contre, il est particulièrement hasardeux de vouloir attribuer ces changements à une

11. Il s'agit, à notre avis, de l'une des meilleures recherches empiriques faites dans lc cadre du programme d'évaluation mis sur pied par le ministère de la Justice du Canada. 
dimension isolée et spécifique de la réforme (par exemple l'abolition de l'infraction de viol et son remplacement par une autre infraction).

En fait, le caractère soutenu de ces augmentations semble difficilement dissociable d'un ensemble d'autres facteurs entourant et accompagnant la réforme. Pensons, par exemple, au rôle joué par les directives adressées à la police et aux procureurs, par l'action éventuelle des groupes de femmes et des centres d'aide, par les tables de concertation entre les différents intervenants sociaux pour faire face à la violence faite aux femmes (Laplante, 1991, p. 8), ou encore par les médias et par la campagne provinciale d'information et de sensibilisation sur la violence (plus tardive). En effet, au Québec, par exemple, le gouvernement a mis sur pied des tables de concertation à travers toutes les régions administratives de la province. Dans la région de l'Outaouais, cette table se composait de 22 membres recrutés dans différents milieux, depuis les salles d'urgence des centres hospitaliers jusqu'aux services d'action communautaire et aux directrices de maison d'hébergement en passant par la Sûreté provinciale, les polices municipales, la Couronne, les C.S.S., les C.L.S.C., le service de probation, etc. (Laplante, 1991, pp. 20-21). Les renseignements disponibles pour le Québec semblent suggérer que la réforme a été appuyée par la mise en place d'un ensemble de dispositifs. Il faut aussi se rappeler que la « mémoire collective » des réformes est relativement courte. Il y a donc peu de chances qu'une simple modification législative provoque de tels effets soutenus s'il n'y a pas de mécanismes visant à l'actualiser.

Par contre, il nous paraît peu vraisemblable de croire que ces «appuis " auraient été mis en place et que cette même augmentation aurait été obtenue sans la réforme de 1983. Car, elle a au moins donné, en surface, un visage modifié du droit criminel en matière d'infractions sexuelles. Lorsque nous parlons ici d'effets ou de conséquences de la réforme, nous ne faisons donc pas exclusivement allusion aux modifications législatives de 1983. Nous considérons plutôt l'ensemble des dimensions et des actions entreprises pour « soutenir » la réforme. Notre pari est cependant que la réforme fait partie intégrante de ce processus global. Or, ces résultats sont en quelque sorte surprenants compte tenu du fait que l'on a sous-estimé jusqu'à présent les effets de la réforme sur le nombre de condamnations et de renvois à la justice criminelle (voir Los, 1990a, p. $171 ; 1990$ b, pp. 33-34).

Il faut noter aussi, même si nous ne pouvons pas présenter ici les données pertinentes (voir Roberts, 1990), que cette augmentation dans le nombre de renvois d'agression sexuelle est beaucoup plus accentuée 
que celle qu'on observe pour les autres infractions contre la personne pour la même période pour le Canada ${ }^{12}$.

Le contexte et les particularités de cette augmentation nous permettent donc d'écarter l'hypothèse selon laquelle cette augmentation statistique serait due à une augmentation dans l'incidence même des agressions sexuelles. En effet, nous savons aujourd'hui que les statistiques pénales ne constituent pas, en règle générale, un bon indicateur empirique de la fréquence des comportements. Elles illustrent plutôt la forme de prise en charge des problèmes sociaux. Or, si cette hypothèse est déjà trop forte concernant d'autres infractions, elle nous paraît particulièrement abusive en ce qui regarde les agressions sexuelles. Car le procès judiciaire est connu ici pour être particulièrement dissuasif pour les victimes. Plus important encore : l'extension, l'intensité et le moment de cette augmentation indiquent - jusqu' où il est possible $\mathrm{d}$ 'indiquer en l'absence d'autres raisons également plausibles en sens contraire - que l'on aurait tort de sous-estimer les effets de la réforme dans son ensemble sur le nombre des renvois.

\section{AUTRES QUESTIONS RELATIVES À L'IMPACT DE LA RÉFORME}

Il y a deux types de statistiques globales relatives aux cas connus par la police : celles relatives aux contentieux considérés comme «fondés " ou "non fondés" et celles concernant le taux d'élucidation par "mise en accusation" (Centre canadien de la statistique juridique, 1988). Les plaintes déclarées par la police comme «non fondées » sont celles dont la "validité " est mise en cause (avant ou après une enquête). L'infraction est supposée ne pas avoir existé. Le taux d'élucidation par mise en accusation désigne, par définition, le pourcentage de cas pour lesquels une personne a été officiellement inculpée même si la police n'a pas appréhendé l'accusé.

D'après les statistiques globales, la réforme n'a pas eu à première vue vraiment d'impact sur aucun de ces deux indicateurs statistiques. Le pourcentage moyen des cas classés comme non fondés pour le Québec dans la période en amont de la réforme est de 6 pour cent et de 7 pour cent pour celle en aval. Pour des raisons que nous ignorons, le

12. Notons, par ailleurs, qu'au Québec l'augmentation des plaintes pour l'ensemble des voies de fait a surpassé (121\% entre 1983 et 1989) celle pour les agressions sexuelles $(90 \%)$. Rappelons que les voies de fait ont bénéficié, en ce qui concerne la violence faite aux femmes, de certains dispositifs mis en place pour les agressions sexuelles. 
pourcentage de cas classés comme non fondés est significativement plus bas au Québec qu'ailleurs au Canada, et cela est vrai dès 1977, alors que ce pourcentage était de 6 pour cent en comparaison de 17 pour cent pour le reste du Canada ${ }^{13}$.

En ce qui concerne le taux de mises en accusation pour le Québec dans la période antérieure à la réforme, un tiers des infractions retenues comme "fondées » sont classées par mises en accusation. Ce pourcentage monte à 49 pour cent dans la post-réforme. Ces deux types de données sont plus élevées que celles trouvées par Baril, Bettez et Viau (1988, p. 96), mais il n'y a pas nécessairement de contradiction, puisque nos données sont pour l'ensemble de la province et pour une période plus longue ${ }^{14}$. Quoi qu'il en soit, il nous semble à présent que cette augmentation ne peut pas être attribuée à la réforme, car il y a eu une augmentation similaire dans le taux d'élucidation de toutes les infractions de violence (Roberts, 1990).

Par rapport aux condamnations, il n'existe pas de banque de données pour le Canada. Les estimations reposent donc uniquement sur les données des recherches sur le terrain. Baril, Bettez et Viau (1988, p. 174) constatent pour Montréal (1982-1985) qu'en général « $67 \%$ des causes se rendant à procès donnent lieu à une condamnation » et qu'en « $20 \%$ des cas l'accusé est acquitté ». Dans 7 pour cent des cas, un accusé a été libéré, pour différentes raisons, sans avoir été pour autant acquitté ${ }^{15}$. Les auteurs ont par ailleurs trouvé une différence dans le taux de condamnations selon la période : il y a eu 74 pour cent de condamnations (plaidoyers et verdicts de culpabilité) pour la période antérieure à la réforme et 58 pour cent pour la période postérieure. Tant les plaidoyers que les verdicts de culpabilité ont diminué. En outre, les acquittements $^{16}$ et les retraits de plaintes ont augmenté. Selon leurs données, il n'est pas possible d'attribuer cette décroissance dans le taux des condamnations à une moindre gravité des contentieux, car ceux-ci

13. En réalité, cette statistique n'est pas la seule par laquelle les données du Québec se distinguent d'autres juridictions au Canada. Le taux de renvoi au Québec est aussi plus bas qu'ailleurs. Par exemple, en 1988, le taux de renvoi (« infractions rélles ») pour les agressions sexuelles au Québec par cent mille habitants était de 57 contre 206 en ColombicBritannique. Les raisons de ces différences n'ont pas encore été élucidées.

14. La recherche de Baril et al. porte sur les années 1981 et 1982 (avant la réforme) et sur les années 1984 et 1985 (après la réforme).

15. Ajoutons que la plainte est retiree dans environ 5 pour cent des cas.

16. À Montréal, les acquittements sont passés de 18 à 24 pour cent pour la période étudiée (Baril, Bettez et Viau, 1989, p. 176). 
présentent peu de changements d'une période à l'autre (p. 176). La question reste donc ouverte.

Malgré l'absence explicite d'une déclaration d'objectifs de la réforme, il est clair qu'un d'eux résidait dans l'augmentation du taux de condamnations (Begin, 1989 ; Chappell, 1984, pp. 73-75; Ministère de la Justice, 1990, p. 52). En effet, comme la réforme avait modifié aussi les critères de la preuve, cette augmentation était attendue. Les recherches évaluatives ont présenté des résultats différents pour chaque ville étudiée, mais on ne trouve, dans l'ensemble, aucune différence importante dans le taux global de condamnations (Ministère de la Justice, 1991, p. 53).

Quoi qu'il en soit, il est important de ne pas confondre ici la réduction ou l'augmentation du taux de condamnations (plaidoyers et verdicts de culpabilité) parmi les causes traduites en procès avec l'augmentation ou la réduction du nombre absolu des condamnations. En effet, l'énorme augmentation dans le nombre de renvois au Canada et au Québec nous permet de penser que le nombre absolu de condamnations a aussi augmenté. En outre, nous savons par les études sur le terrain que les taux d'acquittements n'ont pas augmenté partout (Ministère de la Justice, 1990) et que lorsqu'ils ont augmenté, ils n'arrivent pas à neutraliser l'augmentation dans le nombre des renvois.

Une dernière remarque concernant l'effet de sélection de la justice criminelle. Baril, Bettez et Viau (1988, pp. 82-83) constatent à partir des renseignements disponibles dans les dossiers de police que plus de 85 pour cent des accusés sont sans emploi ( 46 pour cent) ou sont des ouvriers non spécialisés ( 39 pour cent). Les auteures soulignent soigneusement que les valeurs manquantes sont de l'ordre de 60 pour cent, mais leur constat comme tel n'est pas par ailleurs étonnant. En réalité, elles veulent empêcher que l'on pense que les agressions sexuelles sont pratiquées surtout par les hommes des couches sociales défavorisées. Enfin, elles ne trouvent pas de différence statistiquement significative d'une période à l'autre. Le système pénal continue donc à toucher fondamentalement les gens des couches défavorisées.

\section{LA DISTRIBUTION DES AGRESSIONS SEXUELLES DANS LES TROIS NIVEAUX DE GRAVITÉ}

Nous avons constaté ci-dessus que le nombre de renvois à la justice criminelle a augmenté énormément depuis 1983. Nous allons examiner maintenant comment cette augmentation a été ventilée parmi les trois 
niveaux de gravité au fil des sept années qui ont suivi la mise en place de la réforme.

\section{Tableau 4}

La classification des plaintes d'agression sexuelle au Québec (1983-1989)

\begin{tabular}{|c|c|c|c|c|c|c|c|c|}
\hline \multirow{3}{*}{$\begin{array}{l}\text { Années } \\
1983\end{array}$} & \multicolumn{6}{|c|}{ Niveaux de gravité } & \multicolumn{2}{|c|}{ Total } \\
\hline & \multicolumn{2}{|c|}{$\begin{array}{c}\text { Niveau l } \\
\text { (moins grave) }\end{array}$} & \multicolumn{2}{|c|}{ Niveau II } & \multicolumn{2}{|c|}{$\begin{array}{c}\text { Niveau III } \\
\text { (plus grave) }\end{array}$} & & \\
\hline & 1692 & $(81 \%)$ & 227 & $(11 \%)$ & 171 & $(8 \%)$ & 2090 & $(100 \%)$ \\
\hline 1984 & 2105 & $(84 \%)$ & 204 & $(8 \%)$ & 186 & $(7 \%)$ & 2495 & $(100 \%)$ \\
\hline 1985 & 2416 & $(86 \%)$ & 216 & $(8 \%)$ & 178 & $(6 \%)$ & 2810 & $(100 \%)$ \\
\hline 1986 & 2907 & $(89 \%)$ & 215 & $(7 \%)$ & 145 & $(4 \%)$ & 3267 & $(100 \%)$ \\
\hline 1987 & 3125 & $(90 \%)$ & 209 & $(6 \%)$ & 142 & $(4 \%)$ & 3476 & $(100 \%)$ \\
\hline 1988 & 3473 & $(92 \%)$ & 194 & $(5 \%)$ & 111 & $(3 \%)$ & 3778 & $(100 \%)$ \\
\hline 1989 & 3632 & $(91 \%)$ & 209 & $(5 \%)$ & 135 & $(3 \%)$ & 3976 & $(100 \%)$ \\
\hline
\end{tabular}

Source : Données de Statistique Canada.

Un total de 2090 plaintes d'agression sexuelle a été enregistré au Québec en 1983. On constate que 81 pour cent de ce total a été classifié au premier niveau de gravité, 11 pour cent au deuxième niveau et 8 pour cent au troisième niveau. Cependant, cette distribution se modifie progressivement: le premier niveau augmente tellement qu'il représente 90 pour cent (ou plus) de tous les dossiers de leur année respective depuis 1987 . On voit aussi que pour le Québec l'augmentation des plaintes a été entièrement absorbée par le premier niveau. En effet, ces contentieux ont augmenté de 115 pour cent entre 1983 et 1989 (soit de 1692 a 3 632), tandis qu'on accuse une décroissance de 8 pour cent pour le deuxième (soit de 227 à 209) et de 21 pour cent pour le troisième (soit de 171 à 135) niveaux.

Les données pour le Canada sont encore plus éloquentes : en 1983. 88 pour cent des contentieux sont classés au premier niveau, 7 pour cent au deuxième et 5 pour cent au troisième ; en 1989,96 pour cent sont au premier, 3 pour cent au deuxième et 1 pour cent seulement au troisième niveau. L'augmentation a été absorbée entièrement par le premier niveau. En effet, il y a eu 12241 plaintes enregistrées en 1983 et 30340 en 1989 , soit une augmentation de 148 pour cent. On observe aussi une décroissance progressive du nombre et du taux des contentieux au troisième niveau : on compte 685 cas en 1983 et 445 en 1989 , soit 35 pour cent de moins. La seule différence apparente avec le Québec réside dans le deuxième niveau: les chiffres accusent une légère augmentation de 
5 pour cent pour le Canada entre 1983 et 1989 (soit de 925 à 971). Cependant, cette augmentation est compensée par la chute du troisième niveau. On accuse alors une décroissance dans l'ensemble des niveaux II et III.

Tableau 5

La classification des plaintes d'agression sexuelle au Canada (1983-1989)

\begin{tabular}{lcccccc}
\hline Années & \multicolumn{3}{c}{$\begin{array}{c}\text { Niveaux de gravité } \\
\text { Niveau I } \\
\text { (moins grave) }\end{array}$} & Niveau II & $\begin{array}{c}\text { Tiveau III } \\
\text { (plus grave) }\end{array}$ & \\
\hline 1983 & $12241(88 \%)$ & $925(7 \%)$ & $685(5 \%)$ & $13851(100 \%)$ \\
1984 & $15805(91 \%)$ & $878(5 \%)$ & $640(4 \%)$ & $17323(100 \%)$ \\
1985 & $19756(93 \%)$ & $918(4 \%)$ & $590(3 \%)$ & $21264(100 \%)$ \\
1986 & $22623(93 \%)$ & $1001(4 \%)$ & $490(3 \%)$ & $24114(100 \%)$ \\
1987 & $24949(94 \%)$ & $1034(4 \%)$ & $460(2 \%)$ & $26443(100 \%)$ \\
1988 & $27655(95 \%)$ & $1041(4 \%)$ & $415(1 \%)$ & $29111(100 \%)$ \\
1989 & $30340(96 \%)$ & $971(3 \%)$ & $445(1 \%)$ & $31756(100 \%)$ \\
\hline
\end{tabular}

Source : Données de Statistique Canada.

Comment peut-on expliquer cette augmentation dans le nombre de classifications au premier niveau de gravité ? Le lecteur doit garder à l'esprit que les explications proposées ici ont le statut d'interprétations post factum. C'est dire qu'il s'agit d'interprétations qui s'ajustent bien aux données mais qui ne découlent pas à proprement parler directement d'elles (Merton, 1949). Elles ne nous permettent donc pas de trancher, de manière claire et avec un argument empirique supplémentaire, entre diverses autres interpretations rivales pouvant aussi se conformer à ces résultats. Les interprétations sont donc, pour ainsi dire, exploratoires.

À première vue, on pourrait penser qu'il y a eu tout simplement une augmentation dans le nombre de cas «moins graves " qui ont été rapportés à la police. Ceci serait d'ailleurs l'une des conséquences possibles de la réforme législative : l'un des objectifs attribués au projet de loi C-127 était celui de stimuler le renvoi à la police d'un plus grand nombre de cas, et il est raisonnable d'assumer que, dans le passé, la gravité de l'agression était positivement corrélée aux chances que la victime porte plainte à la police. Il faut se rappeler que les procès pour viol ont toujours été hautement « dissuasifs » pour les victimes. On peut aussi supposer que les situations d'agression «les plus violentes » soient aussi les moins répandues et qu'une augmentation importante 
dans le nombre des plaintes va chercher nécessairement dans le bassin des cas moins graves.

Mais il y a au moins deux problèmes avec cette hypothèse. Premièrement, elle est insuffisante puisqu'elle n'explique pas le déclin dans le nombre de cas classifiés au troisième niveau de gravité au Québec et au Canada. Deuxièmement, cette hypothèse est au moins en partie mise en cause par les données (plus restreintes mais plus détaillées) de la recherche de Baril, Bettez et Viau (1988, pp. 61-69). En comparant deux années avant la réforme (1981 et 1983) avec deux années après la réforme (1984 et 1985) à Montréal, ces auteures n'ont trouvé aucune différence remarquable dans la nature des agressions dénoncées à la police.

On peut aussi vouloir expliquer l'augmentation des classifications au premier niveau par une tendance du personnel de la justice à faire une classification «à la baisse » des agressions sexuelles. En effet, on a dit que, par rapport-aux agressions sexuelles, la police a tendance à délaisser les plaintes, à les classer comme non-fondées ou encore à rendre dissuasif pour les victimes le recours à une enquête. Il est donc possible que la police soit en train d'enregistrer et de donner suite à un plus grand nombre de contentieux mais qu'elle soit en train de privilégier le premier niveau de gravité alors qu'elle aurait dû utiliser les autres. Cette pratique aurait pu d'ailleurs être renforcée par le fait que la peine maximale ici est déjà tellement élevée que l'utilisation de cette catégorie laisse quand même une marge de mancuvre assez confortable pour les peines et donc pour la négociation des plaidoyers. Cependant, cette hypothèse ne trouve pas non plus de support dans la recherche de Baril, Bettez et Viau (1988, p. 69). Selon les auteures, "la classification de la police correspond d'assez près aux descriptions contenues dans les rapports ». Certes, elles ont pu constater certains écarts à la baisse. En effet, certaines agressions armées ont été classifiées au premier niveau (p. 69) plutôt qu'au deuxième. Mais ces écarts sont trop petits pour expliquer l'augmentation observée dans les tableaux 4 et 5 .

En outre, les renseignements statistiques sur les infractions de voies de fait ne semblent pas non plus appuyer facilement cette hypothèse d'une classification à la baisse par la police. Il faut se rappeler ici que la réforme des infractions de voies de fait a accompagné celle des agressions sexuelles et que l'on a créé aussi trois niveaux de voies de fait : le premier niveau n'a pas non plus de définition explicite ${ }^{17}$; le

17. Voir note 7 ci-dessus. 
deuxième («agression armée ou infliction de lésions corporelles ») est défini quasiment de la même façon que le deuxième niveau d'agression sexuelle et le troisième niveau, appelé ainsi par analogie aux « voies de fait graves ", reprend mot à mot la même définition du troisième niveau d'agression sexuelle. La seule différence notable entre ces deux groupes d'infractions réside dans les peines maximales. En effet, cellesci sont systématiquement inférieures pour chaque niveau des voies de fait ( 5 ans ; 10 ans ; 14 ans). Nous attirons particulièrement l'attention sur le fait que la peine maximale du premier niveau est de 5 et non de 10 ans comme pour l'agression sexuelle. Cette peine de cinq ans peut stimuler la classification de certains cas au deuxième niveau afin de favoriser la négociation des plaidoyers ou de justifier la demande pour une peine plus élevée. Le tableau 6 présente les données pour le Québec.

Tableau 6

La classification des plaintes de voies de fait au Québec (1983-1989)

\begin{tabular}{|c|c|c|c|c|c|}
\hline \multirow{3}{*}{$\begin{array}{l}\text { Années } \\
1983\end{array}$} & \multicolumn{3}{|c|}{ Niveaux de gravité } & \multicolumn{2}{|c|}{ Total } \\
\hline & $\begin{array}{c}\text { Niveau I } \\
\text { (moins grave) }\end{array}$ & Niveau $I I$ & $\begin{array}{c}\text { Niveau III } \\
\text { (plus grave) }\end{array}$ & & \\
\hline & $9883(75 \%)$ & $2624(20 \%)$ & $707(5 \%)$ & 13214 & $(100 \%)$ \\
\hline 1984 & $11854(76 \%)$ & $3056(20 \%)$ & $617(4 \%)$ & 15527 & $(100 \%)$ \\
\hline 1985 & $13616(78 \%)$ & $3289(19 \%)$ & $483(3 \%)$ & 17388 & $(100 \%)$ \\
\hline 1986 & $15836(78 \%)$ & $3867(19 \%)$ & $512(3 \%)$ & 20215 & $(100 \%)$ \\
\hline 1987 & $19392(80 \%)$ & $4323(18 \%)$ & $499(2 \%)$ & 24214 & $(100 \%)$ \\
\hline 1988 & $21837(80 \%)$ & $4842(18 \%)$ & $606(2 \%)$ & 27285 & $(100 \%)$ \\
\hline 1989 & $23688 \quad(81 \%)$ & $4859(17 \%)$ & $681(2 \%)$ & 29228 & $(100 \%)$ \\
\hline
\end{tabular}

Source : Données de Statistique Canada.

Si l'on compare les tableaux 4 et 6 , on remarque qu'ils se ressemblent sur au moins deux points importants. Tout d'abord, le nombre de plaintes classées au troisième niveau est, toutes proportions gardées, très réduit dans les deux cas. Ensuite, on observe aussi dans les deux tableaux une décroissance dans le nombre absolu de cas classifiés au troisième niveau, bien que cette décroissance commence plus tard (1986) pour les agressions sexuelles. On constate aussi une légère remontée vers la fin de la période. Ceci semble suggérer que ce qui arrive à certains égards aux agressions sexuelles sur le plan de la classification arrive aussi en partie aux voies de fait. Cette question reste cependant ouverte. 
On voit que les voies de fait sont aussi, dans la majorité des cas, classées au premier niveau de gravité. En 1983, seulement 5 pour cent de toutes les plaintes pour voies de fait ont été classées au troisième niveau, contre 8 pour cent pour les agressions sexuelles. En 1989, seulement 2 pour cent de toutes ces plaintes sont au troisième niveau, contre 3 pour cent pour les agressions sexuelles. Et les voies de fait classées au troisième niveau sont passées de 707 en 1983 à 681 en 1989 , soit une décroissance de 4 pour cent. La différence la plus remarquable est que les voies de fait ont été classées dès 1983 dans une proportion plus réduite au troisième niveau. Tout se passe comme si les voies de fait avaient été «aménagées » d'emblée plus facilement à la nouvelle structure tripartite.

Nous observons d'ailleurs, grosso modo, cette même tendance à l'échelle du Canada. En 1983, seulement 3 pour cent de tous les dossiers de voies de fait sont classés au troisième niveau, contre 5 pour cent pour les agressions sexuelles. En 1989, les voies de fait du troisième niveau comptent seulement pour 2 pour cent de l'ensemble des contentieux contre 1 pour cent pour les agressions sexuelles ${ }^{18}$. De même, les voies de fait classées au troisième niveau sont passées de 3641 à 3383 , soit une décroissance de l'ordre de 7 pour cent entre 1983 et 1989 (par opposition à $35 \%$ pour les agressions sexuelles). Ces données ne nous sembient donc pas appuyer l'hypothèse selon laquelle l'augmentation du premier niveau des agressions sexuelles et l'utilisation limitée du troisième niveau s'expliquerait surtout par une tendance de la police (ou de la justice) à classifier à la baisse ces infractions.

Ceci nous amène à envisager une dernière hypothèse qui vise à expliquer surtout la décroissance dans le taux et dans le nombre absolu de cas d'agressions sexuelles classifiées au troisième niveau entre 1983 et 1989. Selon cette hypothèse, les agences de contrôle auraient pris un certain temps pour réorganiser leurs pratiques en fonction des nouvelles infractions sexuelles. Rappelons que le projet de loi C-127 a modifié les critères de classification des infractions sexuelles, car auparavant elle se faisait particulièrement en fonction du critère de la pénétration. Les nouveaux criteres de classification ne portent maintenant que sur le degré de violence, la menace, l'utilisation d'armes, etc. On peut donc s’attendre à une certaine période d'ajustement à ces critères et d'interaction entre les différents paliers de la justice criminelle. Il faut se

18. En réalite, si l'on n'arrondit pas les chiffres, cette différence est encore plus négligeable en $1989: 1,8$ pour cent pour les voies de fait et 1,4 pour cent pour les agressions sexuelles. Notons que le groupe des voies de fait est beaucoup volumineux. 
rappeler à cet égard que les définitions des deux derniers niveaux ne sont pas des plus limpides ${ }^{19}$.

Les données de la recherche de Baril, Bettez et Viau (1988) ne semblent pas mettre en doute cette dernière hypothèse. Les auteures notent que « la plupart (70 pour cent) des agressions rapportées à la police sont de peu de gravité si l'on considère objectivement la nature des actes subis par la victime et décrits au précis de police" (p. 68). L'utilisation d'armes est aussi peu fréquente ( 10 pour cent) et ce. même si les auteures constatent differentes formes de violence physique comme moyen de contrainte dans environ la moitié des cas (p. 69). Il est donc possible que la grande partie de cette violence «visible» puisse entrer "adequatement " dans les deux premières catégories d'agression selon la nouvelle logique du système. C'est dire que les opérateurs ont peut-être de «bonnes raisons" (selon les règles du jeu) pour faire une telle classification. Quoi qu'il en soit, la compréhension plus approfondie de cette question réclame d'autres types de recherches.

\section{UNE ÉVALUATION PRÉLIMINAIRE DES EFFETS PRATIQUES DE LA STRUCTURE TRIPARTITE}

Nous pouvons maintenant nous interroger, dans un autre ordre d'idées, sur la "valeur pratique » de cette structure tripartite. En effet, peut-on dire que cette structure a été utile ou a contribué à cette augmentation observée dans le nombre de renvois et, indirectement, dans le nombre absolu de condamnations ?

Nos données ne nous permettent pas d"apporter une réponse directe à cette question. Cependant, nous inclinons à penser que cette augmentation doit être plutôt attribuée à la réforme dans son ensemble qu'à la structure tripartite elle-même. Rappelons à cet égard que le taux des condamnations par rapport au nombre de mises en accusation est demeuré, en général, sensiblement le même (Ministère de la Justice, 1990). Au Québec, ce taux a même accusé une décroissance (Baril, Bettez et Viau, 1988). Certes, il y a fort probablement eu une augmentation du nombre absolu de condamnations, mais cela découle de l'augmentation marquée dans le nombre de renvois au système. En outre, les autres statistiques disponibles sur les plaintes «fondées » et « non fondées », sur les taux de mise en accusation, etc., n'ont pas subi non plus

19. Dans le passé, la classification des infractions pour voies de fait se faisait déjà en fonction du degré de violence. La modification dans les critères est donc un peu moins marquee dans ces cas-ci. 
de modifications significatives après la réforme. De plus, il est fort probable que les trois nouvelles infractions, avec leurs definitions confuses (ou leur absence de définition), aient plutôt contribué à créer un imbroglio pour les tribunaux. Il est donc fort probable que même la première proposition de la Commission de réforme visant à créer une seule infraction aurait pratiquement eu le même résultat.

\section{L'AMBIGUÏTÉ SYMBOLIQUE DE LA NOUVELLE STRUCTURE TRIPARTITE}

Que cette nouvelle structure ait eu ou non des effets pratiques sur le nombre de renvois et de condamnations, une chose est certaine : elle a créé une énorme ambiguité symbolique autour de ces infractions, et cette ambiguiité peut, à son tour, avoir des conséquences sociales négatives. Pour comprendre cette ambiguïté et ses conséquences pratiques possibles, il faut récapituler le cheminement conceptuel de la réforme.

Comme nous l'avons souligné, les trois ou quatre anciennes infractions de viol, tentative de viol et attentat à la pudeur ne véhiculaient que discrètement l'idée d'une échelle de gravité. La raison en est que cette image ne pouvait être saisie que par les peines maximales, car chaque infraction était conçue comme relevant d'un type différent (voir sections 1 et 2). Une autre caractéristique de l'ancienne structure est qu'elle désignait, toutes proportions gardées, d'une manière moins confuse ce qui devait entrer dans chaque catégorie. Bien sûr, on pouvait, par exemple, critiquer la notion de viol parce qu'elle ne protégeait pas les femmes mariées contre leurs conjoints ou parce qu'elle se limitait à la pénétration vaginale (C.R.D.C.. 1978b, p. 14). Mais on pouvait au moins savoir grosso modo ce que la loi considérait comme un viol, une tentative de viol et (moins clairement) un attentat à la pudeur.

Illustrons le problème à l'aide d'un autre exemple. Supposons qu'il $y$ ait dans le code une infraction de " vol " et une autre de "vol à main armée ». On peut sans doute mettre en doute la pertinence de l'une ou l'autre de ces catégories et soutenir qu'elles devraient être fusionnées dans une seule infraction ou que le "vol à main armée " devrait avoir une définition plus restrictive pour exclure, par exemple, le cas des armes simulées. On peut aussi dénoncer les cas de "vols à main armée " qui sont classés comme "vol " et vice versa. Mais l'énoncé ou la demande suivante ne ferait aucun sens : «il faut classer plus de cas de vol dans la catégorie des vols à main armée pour montrer que nous réprouvons moralement le vol ". 
De même, avant la réforme de 1983, très peu de chercheurs seraient tentés de comparer le nombre de contentieux classifiés dans chacune des trois anciennes catégories et dire ceci: «il y a très peu de cas classifiés comme viols ; il serait souhaitable que l'on classifie comme viols un certain nombre d'attentats à la pudeur pour montrer symboliquement que nous désapprouvons fortement cet ensemble de comportements ». Bien sûr, on pouvait dire, mais cela est un énoncé différent, "que la notion de viol a été définie de façon excessivement étroite», puisqu'elle mettait l'accent sur la pénétration vaginale et excluait les femmes mariées. Le chercheur pouvait aussi dénoncer une classification injustifiée : par exemple, un cas de viol qui aurait été classé comme attentat à la pudeur, etc. Mais la "communication symbolique " entre ces diverses catégories n'était pas aussi ouverte qu'elle l'est maintenant. Aujourd'hui, les critères de classification ne sont plus aussi évidents et nous pouvons désormais tenir un discours qui regrette le fait qu'il y ait « très peu de cas classifiés aux niveaux II et III ».

Bien sûr, la première proposition de la Commission de réforme pour créer une infraction unique aurait eu pour résultat d'éliminer radicalement ce problème de classification, puisqu'il n'y aurait qu'une seule catégorie d'agression sexuelle. La gravité ne serait prise en ligne de compte qu'au moment de la détermination de la peine.

La deuxième proposition de la Commission de réforme réintroduit explicitement la représentation d'une échelle de gravité, mais elle le fait "à la manière ancienne", c'est-à-dire sous la forme de deux "espèces" de comportements différents : une infraction d'attouchement sexuel (sans menace et sans violence physique) et l'autre d'agression sexuelle (avec menace et violence physique). Ces deux catégories semblent alors moins ouvertes symboliquement, et peut-être aussi plus clairement définies, que celles en vigueur.

Mais qu'est-ce qui se passe dans le cas de la nouvelle structure tripartite retenue par la loi ? Cette structure projette la pleine image d'une échelle de gravité où les critères d'inclusion et d'exclusion dans chaque niveau ne sont pas tout à fait clairs. La caractéristique principale de cette structure est qu'elle a sapé fondamentalement la représentation d'un cloisonnement entre les trois (niveaux d')infractions, maximalisant la «communication symbolique » et, éventuellement, la possibilité d'un flux de dossiers entre celles-ci. Elle a transformé alors, du point de vue des représentations, les trois anciennes infractions en une seule infraction divisée en trois niveaux de gravité tous très mal définis. C'est seulement dans un sens strictement technique que continuent à exister 
" trois infractions". Le résultat est qu'on peut maintenant regretter le fait "d'avoir très peu de cas classifiés au niveaux II et III de gravité ", puisqu'on a désormais l'impression que le critère de classification est devenu davantage un jugement sur la gravité générale de ces comportements plutôt qu'un jugement sur des variations déterminées de ces comportements.

D'où le résultat paradoxal suivant: on a refusé la proposition visant à créer une infraction unique, mais on a crée, au bout du compte, sur le plan des représentations, l'infraction unique que l'on avait rejetée.

Mais le paradoxe ne s'arrête pas là. Le «législateur » a tellement voulu montrer, par le biais de peines maximales exorbitantes, que même la forme la moins grave de "cette infraction " était extrêmement grave qu'il a fini par montrer symboliquement l'inverse : que le système de justice est en train de classifier, dans la pratique, quasiment tous les cas ( $96 \%$ au Canada) au niveau le moins grave de l'échelle de gravité. La nouvelle loi a créé alors les conditions pour la production d'un « effet symbolique renversé ». En effet, on peut maintenant lire les statistiques pénales de la manière suivante: " la police ou les tribunaux considèrent la grande majorité des cas comme très simples, insuffisamment sérieux pour mériter d'être classés au niveau II ou III ».

Dans le passé, lorsqu'on a discuté l'idée d'abandonner la notion juridique de "viol " pour la remplacer par celle d'agression sexuelle, quelques chercheurs œuvrant dans une perspective féministe craignaient que cette modification en vienne à banaliser le viol. Il nous paraît que la nouvelle structure tripartite en elle-même est en train d'y contribuer d'une nouvelle manière.

Le problème ici est qu'on doit admettre qu'il n'est pas clair encore que ce soit la loi dans sa totalité qui banalise le viol parce que, entre autres choses, la loi a augmenté dramatiquement les peines maximales pour les deux infractions moins graves. Mais la loi ouvre la possibilité, sous un autre angle, de considérer le viol comme banalisé par la justice criminelle. Et si cela est le cas, un autre paradoxe apparaît ici : c'est que la nouvelle loi a banalisé le viol en partie parce qu'elle a survalorisé, en termes de peine maximale, l'ancienne infraction d'attentat à la pudeur, en l'occurrence les formes moins graves d'agression sexuelle.

Baril, Bettez et Viau $(1988$, p. 61) ont pressenti cet effet symbolique renversé lorsqu'elles ont constaté que 84 pour cent des agressions sexuelles pour 1984 et 1985 avaient été classifiées au premier niveau. 
Cela leur a paru confirmer la crainte d'une "banalisation du viol" exprimée par certains groupes. En réalité, ces derniers faisaient référence à l'abandon même de la notion légale de viol et non à la classification des plaintes. Cependant, nous croyons que la nouvelle loi a crée des conditions pour qu' on y voit aussi une nouvelle manière de banaliser le viol.

Rappelons que la nouvelle loi utilise l'expression «agression sexuelle grave " pour qualifier justement le niveau III qui correspond a 1 pour cent des cas au Canada. Or, ce qualificatif était absent dans les propositions précédentes. La loi se garde bien de qualifier les deux autres niveaux et $\mathbf{n}$ 'utilise surtout pas l'expression « agression sexuelle simple " pour désigner le premier niveau. Il serait d'ailleurs incongru de qualifier une infraction de "simple " et de prévoir en même temps une peine maximale de dix ans. Mais l'ambiguïté est créé de toute façon, d'autant que la loi est muette sur la manière d'appeler et de définir le premier niveau. Nous nous sommes rendu compte qu'on a déjà commencé à désigner le premier niveau d'agression sexuelle par l'expression "agression sexuelle simple " tant au niveau de la pratique policière, des documents officiels (Ministère de la Justice, 1990, p. 15) que de la recherche. En effet, même des auteures averties l'ont employée (Baril, Bettez et Viau. 1988, pp. 53, 60, 185). Selon toute vraisemblance, il s'agit ici d'une transposition, au niveau du langage, de l'ancienne infraction de « voies de fait». En effet, avant la réforme de 1983, il existait dans le code, dans sa version française, une infraction de «voies de fait simple» (art. 245, (1)). Mais il s'agissait là d'une infraction conçue comme vraiment simple : avec une poursuite sur déclaration sommaire de culpabilité (peine maximale : 6 mois et/ou amende).

Une dernière remarque. L'effet symbolique renversé créé par la nouvelle structure tripartite risque par ailleurs d'avoir des conséquences négatives, c'est-à-dire de créer une «inflation » dans l'exercice de la pénalité. Car ce constat d'une «banalisation» du viol par une classification à la baisse peut justement donner lieu à un discours qui déplore le grand nombre de contentieux au premier niveau de gravité, faisant ainsi indirectement des pressions sur les tribunaux pour une plus grande utilisation des niveaux II et III. Pour mieux comprendre les enjeux ici, il faut apporter un éclaircissement sur la notion de gravité dans la logique punitive de ce droit criminel que nous avons hérité du xville siècle. Car, sous un certain angle, la représentation de la gravité dans le champ pénal s'écarte considérablement de la représentation de 
la gravité dans le champ du sens ordinaire, et ce même si une distinction nette entre ces deux champs n'est ni aisée ni tranchée.

En premier lieu, la représentation de la gravité dans le champ pénal a un point de référence et un arrière-plan propres. Par exemple, pour une victime, la gravité est déterminée fondamentalement par son expérience vécue. Par contre, le système déplace en bonne partie ce point de référence. Car, dans l'exercice de l'activité classificatrice et répressive, l'idéologie pénale "amène " le personnel de la justice à faire des comparaisons entre différentes infractions, victimes, circonstances, etc., en vue d'une intervention éventuelle. Résultat: ce qui est vécu comme «grave»par la victime peut être perçu comme «comparativement moins grave" par le système compte tenu d'autres histoires de vie, circonstances, etc., et des «mesures répressives " que le système est susceptible de déployer. Ceci constitue l'une des formes par lesquelles le système pénal est susceptible de disqualifier l'expérience des victimes $^{20}$. Dans le cas des agressions sexuelles, et compte tenu des rapports de pouvoir entre les hommes et les femmes, ce peut être très éprouvant pour ces dernières. Or, une classification tripartite vague avec des peines élevées à tous les niveaux et mettant l'accent sur une "pure " échelle de gravité augmente considérablement les chances d'une disqualification de l'expérience des victimes.

En deuxième lieu, la notion de gravité a une visée pratique particulière dans l'idéologie pénale. Par exemple, lorsque le sens ordinaire définit une situation comme grave, il exprime sa préoccupation avec cette situation ou son degré de réprobation morale. Par là, on vise pratiquement souvent beaucoup plus à obtenir une forme quelconque de reconnaissance. En outre, comme le « pouvoir de punir» nous échappe dans la vie quotidienne ou prend des formes plus diffuses, indirectes et ponctuelles, le discours du sens ordinaire sur la gravité est, pour ainsi dire, "dégagé " de ses rapports avec l'exercice de la répression. Par contre, l'évaluation de la gravité dans le champ pénal est, dès le départ, moins un jugement moral qu'un jugement pratique, et la fonction de ce jugement est de décider institutionnellement du cas et de «calibrer » la pénalité. C'est dire que la notion de gravité joue, dans le système, un double rôle : celui de "frein » ou celui d' « accélérateur » par rapport à la punition. L'un des problèmes ici est que notre système pénal n'est pas (encore) capable d’agir de la même manière que les personnes peuvent le faire dans leur vie quotidienne : le système est incapable

20. Pour une analyse approfondie d'autres formes de disqualification, voir Smart (1989). 


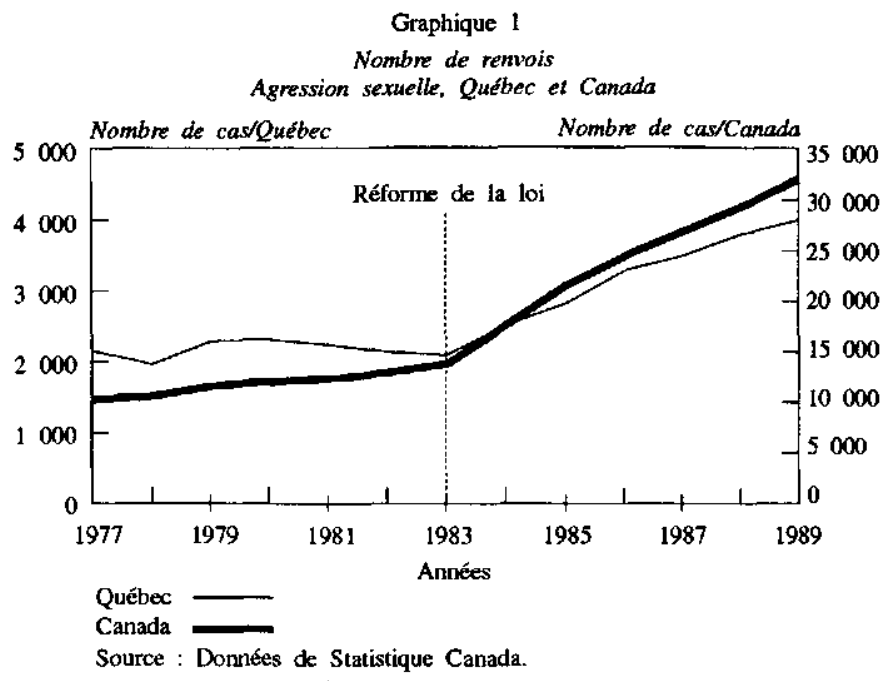

d'exprimer une forte indignation morale sans punir d'une manière sauvage ; s'il met l'accent sur l'indignation, il tend aussi à réprimer plus sévèrement et il est encore caractérisé par une structure de peines vieille et inflationniste. C'est dire que si nous cherchons dans le systèrne actuel la reconnaissance du blâme, nous promouvons en même temps la répression. Mais le système crée aussi une difficulté pour ceux qui favorisent une politique pénale modérée. En effet, les personnes intéressées par l'affirmation du principe de modération sont dans une position très délicate, voire conflictuelle, parce qu'une des manières $\mathrm{d}^{\prime} \mathrm{y}$ parvenir et de s'assurer que le message sera reçu par le système est de minimiser la "gravité » de certains cas que l'on considère. par ailleurs, comme un véritable problème moral ou social. Il faut alors, en permanence, affirmer simultanément la dimension sexiste et agressive de ces situations et la nécessité, malgré cela, de trouver des formes moins répressives d'intervention.

Les rédacteurs du projet de loi C- 127 avaient sans doute une vision relativement claire des problèmes que soulevait l'ancienne loi sur le viol. Les nouvelles infractions sur les agressions sexuelles en ont redressé plusieurs : biais sexistes consacrés par la loi, importance accordée à la pénétration vaginale, etc. Cependant, en créant la nouvelle structure tripartite avec ses peines maximales respectives, les rédacteurs du projet ont créé de nouveaux problèmes. Le défi maintenant semble être de les corriger sans poser d'autres problèmes du même genre et de 
manière à permettre une prise en charge adéquate et juste des agressions sexuelles.

Bien sûr, on peut se demander avec Smart $(1989$, p. 5) dans quelle mesure le droit, et tout particulièrement le droit criminel actuel, peut vraiment accomplir une telle tâche. En ce qui nous concerne, un système de justice criminelle axé surtout sur les principes de la dissuasion par la sévérité des peines, de la rétribution ou de la punition ne constitue pas une manière adéquate de traiter cet important problème social.

\section{BIBLIOGRAPHIE}

BARIL, M., BETTEZ, M.-J. et VIAU, L. (1988), Les Agressions sexuelles avant et après la réforme de 1983: Une évaluation des pratiques dans le district judiciaire de Montréal, Ottawa, Ministère de la Justice du Canada.

BEGIN, P. (1989), « Rape Law Reform in Canada : Evaluating Impact », in E. Viano (sous la direction de): Crime and its Victims: International and Public Policy Issues, New York, Hemisphere Publishing.

BELL, V. (1991), "Beyond the "Thorny Question": Feminism, Foucault and the Desexualisation of Rape ", International Journal of Sociology of Law, vol. 19, pp. 83-100.

BOYD, S. et SHEEHY, E. (1986), « Feminist Perspectives on Law : Canadian Theory and Practice ", Canadian Journal of Women and the Law, vol. 2, pp. 1-52.

BOYLE, C. (1984), Sexual Assault, Toronto, Carswell.

CAMPBELL, D. T. (1969), "Reforms as Experiments $"$, in C. H. Weiss (sous la direction de), Evaluating Actions Programs, Boston, Allyn \& Bacon, Inc.

CANADIAN CENTRE FOR JUSTICE STATISTICS (1988), Excerpts from the Uniform Crime Reporting Manual, Ottawa, Canadian Centre for Justice Statistics, Statistics Canada.

CHAPPELL, D. (1984), « The Impact of Rape Legislation Reform : Some Comparative Trends ", International Journal of Women's Studies, $\mathrm{n}^{\circ}$ 7, pp. 70-80.

CHASE, G. (1983), "An Analysis of the New Sexual Assault Laws", Canadian Women's Studies, $\mathrm{n}^{\circ} 4$, pp. 53-54.

CLARK, L. et LEWIS, D. (1977), Rape. The Price of Coercive Sexuality, Toronto, The Women's Press.

COHEN, L. et BACKHOUSE, C. (1983), « Desexualizing Rape, Dissenting View on the Proposed Rape Amendments ", Canadian Women's Studies, $n^{\circ} 4$, pp. 53-54. 
COMMISSION CANADIENNE SUR LA DÉTERMINATION DE LA PEINE (1987), Réformer la sentence, Ottawa, Ministre des Approvisionnements et Services Canada.

COMMISSION DE RÉFORME DU DROIT DU CANADA (1978a), Infractions sexuelles (Document de travail 22), Ottawa, Ministre des Approvisionnements et Services Canada.

COMMISSION DE REFORME DU DROIT DU CANADA (1978b), Les Infractions sexuelles (Rapport), Ottawa, Ministre des Approvisionnements et Services Canada.

COMMISSION DE RÉFORME DU DROIT DU CANADA (1984), Voies de fait (Document de travail $n^{\circ} 38$ ), Ottawa, Ministre des Approvisionnements et Services Canada.

DAWSON, B. (1987), "Sexual Assault Law and Past Sexual Conduct of the Primary Witness : the Construction of Relevance $»$, Canadian Journal of Women and the Law, $\mathrm{n}^{\circ} 2$, pp. $310-334$.

GRIFFIN, S. (1971), « Rape : All-American Crime », Ramparts, $\mathrm{n}^{\circ} 10$, pp. 26-32,

HINCH, R. (1985), « Canada's New Sexual Assault Laws : A Step Forward for Women ? , Contemporary Crises, $n^{\circ} 9$, pp. 33-44.

HINCH, R. (1988), «Inconsistencies and Contradictions in Canada's Sexual Assault Law ", Canadian Public Policy, vol. 14, pp. 282-294.

LAPLANTE, J. (1991), «La question de la violence familiale et de son traitement ", rapport présenté à la ${ }^{\text {re }}$ Conférence internationale du GREPO, Ottawa, département de criminologie, Université d'Ottawa.

LOS, M. (1990a), * The Struggle over the Definition of Rape in Canada in the 1980s ", Ottawa, Department of Criminology, University of Ottawa.

LOS, M. (1990b), « Feminism and Rape Law Reform », in L. GELSTHORPE et A. MORRIS (sous la dinection de) : Feminist Perspectives in Criminology, Philadelphie, Open University Press.

MacDONALD, D. (1982a), Rape and Consent - The Defence of Mistake of Fact, Ottawa, Standing Committee on Legal and Constitutional Affaits, Library of Parliament.

MacDONALD, D. (1982b), The Evolution of Bill C-127, Ottawa, Standing Committee on Legal and Constitutional Affairs, Library of Parliament.

MERTON, R. K. (1949), Social Theory and Social Structure, New York, The Free Press.

MINISTÈRE DE LA JUSTICE DU CANADA (1990), La Loi sur les agressions sexuelles au Canada. Une évaluation. Vue d'ensemble, Ottawa, Section de la recherche, rapport $n^{\circ} 5$, Ministère de la Justice du Canada.

MINCH, C., LINDEN, R. et JOHNSON, S. (1987), "Attrition in the Processing of Rape Cases », Canadian Journal of Criminology, vol. 29, pp. 389-404. 
PICKARD, T. (1980), "Culpable Mistakes and Rape : Harsh Words on Pappajohn », University of Toronto Law Journal, $\mathrm{n}^{\circ} 30, \mathrm{pp} .15-42$.

PITCH, T. (1985a), « Critical Criminology, the Construction of Social Problems, and the Question of Rape , International Journal of the Sociology of Law, vol. 13, pp. 35-46.

PITCH, T. (1985B), "Violence sexuelle, mouvement féministe, et criminologie critique ", Déviance et Société, vol. 9, n³, pp. 257-265.

RENNER, E. et SAHJPAUL, S. (1986), "The New Sexual Assault Law : What has been its Effect? „, Canadian Journal of Criminology, vol. 28, pp. 407-413.

ROBERTS, J. (1990), La Loi sur les agressions sexuelles au Canada. Une évaluation. Analyse des statistiques nationales, Ottawa : Section de la recherche, rapport $n^{\circ} 4$, Ministère de la Justice du Canada.

RUSSELL, D. (1975), The Politics of Rape, New York, Stein \& Day.

SHEEHY, E. (1987), Autonomie personnelle et droit criminel : quelques questions $d$ 'avenir pour les femmes, Ottawa, Conscil consultatif canadien sur le statut de la femme.

SMART, C. (1989), Feminism and the Power of Law, Londres, Routledge.

SNIDER, L. (1985), "Legal Reform and Social Control : The Dangers of Abolishing Rape », International Journal of the Sociology of Law, vol. 13, pp. 337-356. 